\title{
Cardiac contraction induces discordant alternans and localized block
}

\author{
M. Radszuweit, ${ }^{1}$ E. Alvarez-Lacalle, ${ }^{2}$ M. Bär, ${ }^{3}$ and B. Echebarria ${ }^{2}$ \\ ${ }^{1}$ Weierstrass Institute for Applied Analysis and Stochastics Mohrenstrasse 39, 10117 Berlin, Germany \\ ${ }^{2}$ Departament de Física Aplicada, Universitat Politècnica de Catalunya.BarcelonaTech, Av. Dr. Marañón 44-50, E-08028, Barcelona, Spain \\ ${ }^{3}$ Physikalisch-Technische Bundesanstalt, 10587 Berlin, Germany
}

(Received 7 January 2014; published 4 February 2015)

\begin{abstract}
In this paper we use a simplified model of cardiac excitation-contraction coupling to study the effect of tissue deformation on the dynamics of alternans, i.e., alternations in the duration of the cardiac action potential, that occur at fast pacing rates and are known to be proarrhythmic. We show that small stretch-activated currents can produce large effects and cause a transition from in-phase to off-phase alternations (i.e., from concordant to discordant alternans) and to conduction blocks. We demonstrate numerically and analytically that this effect is the result of a generic change in the slope of the conduction velocity restitution curve due to electromechanical coupling. Thus, excitation-contraction coupling can potentially play a relevant role in the transition to reentry and fibrillation.
\end{abstract}

DOI: 10.1103/PhysRevE.91.022703

PACS number(s): 87.19.Hh, 05.45.-a, 87.19.R-

\section{INTRODUCTION}

The origin of ventricular fibrillation, a life-threatening arrhythmia, lies in the formation and destabilization of reentrant waves of electrical activity. During normal sinus rhythm, the cell transmembrane potential propagates in the form of a pulse-shaped wave, triggering the contraction of the heart. The initiation of reentry often occurs as the wave encounters refractory tissue, thereby resulting in localized block, wave break, and the formation of reentrant spiral waves.

Cardiac alternans is a crucial factor in the emergence of wave breaks [1]. At the single cell level, alternans is characterized by a beat-to-beat alternation in the duration of the excited phase of the cardiac action potential. When propagating along tissue, this may result in spatially homogeneous patterns of oscillations (concordant alternans), or in domains of out-ofphase oscillations (discordant alternans) [2], as the nonlinear wave changes its width as it propagates [3,4]. Strikingly, the main characteristics of this instability can be captured considering a mesoscopic approach [5]. A description in terms of coupled maps, relating the action potential duration (APD) and the conduction velocity $(\mathrm{CV})$ of the action potential, at a given point, with the local time lapse between the end of an excitation and the beginning of the following one [diastolic interval (DI)], reproduces the main characteristic observed during cardiac alternans. Furthermore, close to the transition to alternans, the small oscillations in the APD have been shown to obey a Ginzburg-Landau-type equation with an additional nonlocal term that causes spontaneous nucleations of domains giving rise to discordant alternans [6,7].

The dependencies of APD and conduction velocity on DI are known as APD and CV restitution curves, respectively. They can be easily calculated numerically from simulations of ionic models or measured in experiments. In the simplest formulation, the onset of alternans occurs when the slope of the APD restitution curve is larger than one, giving rise to a period-doubling instability of the underlying periodic rhythm $[8,9]$. While the onset of alternans is determined by the APD restitution, its phenomenology is strongly influenced by the $\mathrm{CV}$ restitution [3,4]. When the slope of the $\mathrm{CV}$ restitution curve is positive and different from zero at the onset of alternans, the transition is always to discordant alternans, with a distance between out-of-phase domains inversely proportional to this slope [6].

This phenomenological formulation has been extended to include such effects as memory [10], intracellular calcium dynamics [11], and nonmonotonic APD [12] or CV restitution curves [13]. In this paper, we consider an effect that is usually neglected when studying the stability of cardiac waves: the contraction of the tissue. This is often considered to be a passive consequence of electrical activity. However, simulations of simplified models of excitation-contraction coupling [14], as well as of reaction-diffusion equations in a medium with a varying (oscillatory) metric [15], suggest that mechanical deformation play an important role for, e.g., the stability of spiral waves. This is due to mechanoelectric feedback whereby mechanical deformations (e.g., stretch) can modulate electric activity [16]. Mechanoelectric feedback is mediated by stretch-activated channels in the cellular membrane [17]. The main goal of this paper is to explore the possibility that contraction acts as a proarrhythmic substrate through the induction of discordant alternans and localized block. For this, we present a mechanism that explains how a small stretch activated current (SAC) can produce a large macroscopic effect.

The paper is organized as follows: in Sec. II we present the ionic model, incorporating the effect of mechanoelectric feedback. In Sec. III we compute its restitution curves, that are used in Sec. IV to derive coupled maps for the dynamics of the APD and DI. With these we check the appearance of discordant alternans as a function of the strength of the stretch activated current and the length of the system. To calculate the minimum tissue size necessary for the onset of discordant alternans, we derive amplitude equations in Sec. V and compute their solutions analytical and numerically. Finally, in the last section we present the conclusions and in the appendixes some more technical details of the derivations.

\section{SIMPLIFIED TISSUE MODEL WITH MECHANOELECTRIC FEEDBACK (MEF)}

The details of the interplay between excitation and contraction are very complex and involve the dynamics of transmembrane potential, ionic currents, intracellular 
calcium concentration, stretch activated currents, and strains and stresses of fibers [18]. To gain insight into the possible proarrhythmic effects of mechanoelectric feedback, an alternative approach is to consider simple models which contain its basic dynamical ingredients [19]. In this spirit, we use a reduced mechanoelectrical model, introduced in Ref. [14], that considers a three-variable Fenton-Karma model [20] for electrical activation, wherein active stress is coupled directly to the transmembrane potential. As proposed in Ref. [21], a linear version of the elastic equations is solved to give rise to a global coupling term in the reaction-diffusion equations. The resulting model reads:

$$
\frac{\partial u}{\partial t}=D \Delta u-J_{f i}(u, v)-J_{s o}(u)-J_{s i}(u, w)-J_{s a}\left(u, T_{a}\right),
$$

$$
\begin{gathered}
\frac{\partial v}{\partial t}=\Theta\left(u_{c}-u\right)(1-v) / \tau_{v}^{-}(u)-\Theta\left(u-u_{c}\right) v / \tau_{v}^{+}, \\
\frac{\partial w}{\partial t}=\Theta\left(u_{c}-u\right)(1-w) / \tau_{w}^{-}-\Theta\left(u-u_{c}\right) w / \tau_{w}^{+}, \\
\frac{\partial T_{a}}{\partial t}=\frac{1}{\tau_{T}(u)}\left(u-T_{a}\right),
\end{gathered}
$$

with relaxation times $\tau_{v}^{-}(u)=\Theta\left(u-u_{v}\right) \tau_{v 1}^{-}+\Theta\left(u_{v}-u\right) \tau_{v 2}^{-}$, $\tau_{T}(u)=\Theta\left(u-u_{T}\right) \tau_{T 1}+\Theta\left(u_{T}-u\right) \tau_{T 2}$, and the currents

$$
\begin{aligned}
J_{f i}(u, v) & =-\frac{v}{\tau_{d}} \Theta\left(u-u_{c}\right)(1-u)\left(u-u_{c}\right), \\
J_{s o}(u) & =\frac{u}{\tau_{0}} \Theta\left(u_{c}-u\right)+\frac{1}{\tau_{r}} \Theta\left(u-u_{c}\right), \\
J_{s i}(u, w) & =-\frac{w}{2 \tau_{s i}}\left\{1+\tanh \left[k\left(u-u_{c}^{s i}\right)\right]\right\}, \\
J_{s a}\left(u, T_{a}\right) & =g_{s}\left(\left\langle T_{a}\right\rangle-T_{a}\right) \Theta\left(\left\langle T_{a}\right\rangle-T_{a}\right)\left(u-E_{s}\right),
\end{aligned}
$$

where $\Theta(\cdot)$ is the Heaviside unit step function, and the constants $g_{s}$ and $E_{s}$ give the conductance and Nernst potential of the stretch activated current $J_{s a}$ [22]. The variable $u$ is a normalized voltage $u=\left(V-V_{0}\right) /\left(V_{f i}-V_{0}\right), T_{a}$ the active tension, and $\left\langle T_{a}\right\rangle=\left[\int_{0}^{L} T_{a}(x) d x\right] / L$ the average active tension along tissue. Thus, the stretch activated current (SAC) is different from zero only in those regions where the local active tension is lower than the average value. The other three currents, a slow inward $\left(J_{s i}\right)$, a slow outward $\left(J_{s o}\right)$, and a fast inward current $\left(J_{f i}\right)$, involve characteristic time constants $\tau_{s i}$, $\tau_{0}, \tau_{d}$ and characteristic activation voltages $u_{c}^{s i}, u_{c}$ [23].

We simulate this model on a one-dimensional (1D) cable of length $L$ with fixed boundaries and nonflux boundary conditions for the nondimensional transmembrane potential $u$. Two sets of parameter values have been selected for the model. A first set corresponds to parameters that fit the restitution curves of the Beeler-Reuter model [20,23,24] (original Beeler-Reuter parameters), which are known to give rise to discordant alternans due to $\mathrm{CV}$ restitution. Alternatively, we have also used a model version where we have modified one of the parameters (the recovery time of the $w$ gate), which is set to $\tau_{w}^{-}=95 \mathrm{~ms}$, instead of the original value $\tau_{w}^{-}=41 \mathrm{~ms}$. This change makes the onset of alternans to appear in a regime
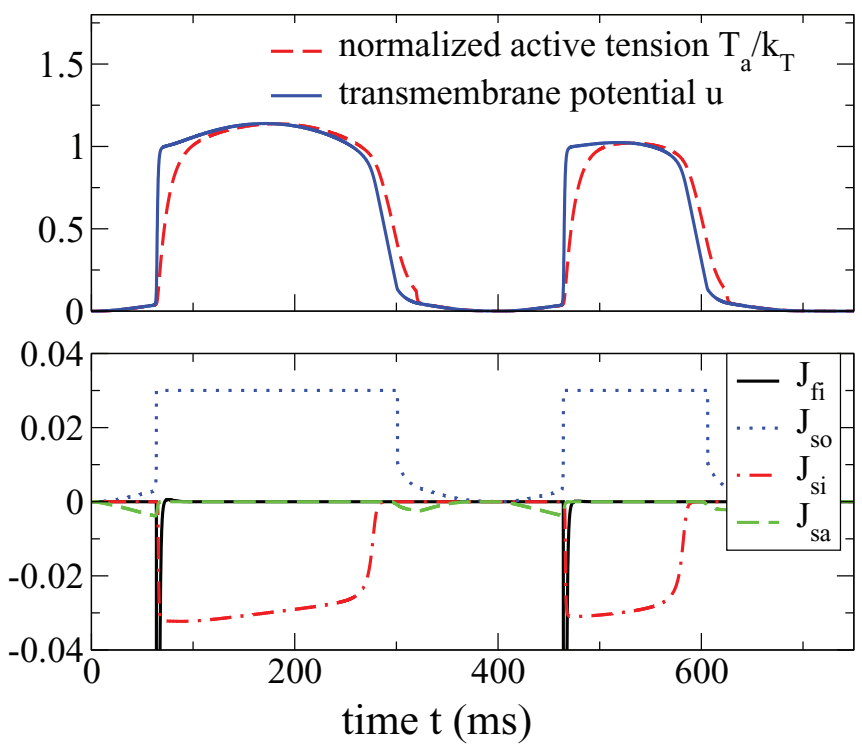

FIG. 1. (Color online) Simulations of Eqs. (1)-(4) for the parameters of the modified Beeler-Reuter model, in a fiber of length $L=$ $6 \mathrm{~cm}$ and with a stretch activated current conductance $g_{s}=0.01 \mathrm{~ms}^{-1}$. (a) Transmembrane potential (solid blue line) and normalized active tension $T_{a} / k_{T}$ (dashed red line). b) Fast inward (solid black), slow outward (dotted blue), slow inward (dot-dashed red), and stretch activated (dashed green) currents.

where the CV restitution curve is flat, so one would not expect to obtain discordant alternans in this case. We will denote modified Beeler-Reuter to this set of parameters. In Fig. 1 we show the transmembrane potential, active tension and the different currents for this set of parameters.

In Fig. 2 we show plots of the spatial distribution of APD obtained with the Beeler-Reuter parameters. Below a given critical fiber length, in the absence of stretch-activated current $\left(g_{s}=0\right)$, concordant alternans appears [see Fig. 2(a)]. However, if we include contraction and increase the strength of the stretch-activated current by a small amount, a transition to discordant alternans is observed for otherwise identical model parameters [Fig. 2(b)]. Thus, small changes in the SAC suffice to transform the characteristics of alternans from concordant to discordant. This finding provokes the question whether this proarrhythmic scenario is a general feature of action potential propagation with electromechanical coupling provided by a stretch activated current.

\section{RESTITUTION MAPS}

We can understand the effect of SAC uncovered in the previous section within the framework of the restitution maps [5]. The change in a single parameter, from the Beeler-Reuter model to the modified Beeler-Reuter model, modifies the APD restitution curve, but not the $\mathrm{CV}$ restitution [Figs. 3(a) and 3(b)], so at the onset of alternans the CV restitution curve is flatter in the latter case than in the former one. In the original parameters the slope of the APD restitution curve becomes larger than one at a diastolic internal of $\mathrm{DI}_{c}=117.2 \mathrm{~ms}$, while for the modified parameters this value is increased to $\mathrm{DI}_{c}=202.4 \mathrm{~ms}$ where the $\mathrm{CV}$ restitution curve is almost flat. 
(a) ||I||||||||

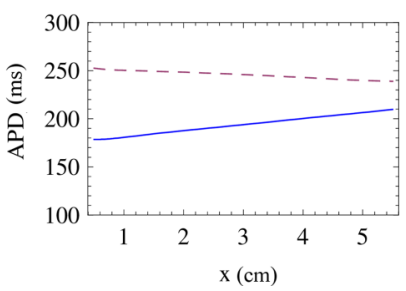

(b)
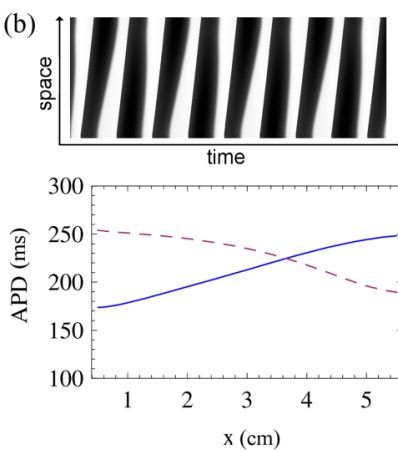

FIG. 2. (Color online) Spatiotemporal plots of the transmembrane voltage (top panels) and APD for two consecutive beats in a cable as a function of the position on the cable (bottom panels, one beat in blue and the next one in dashed red), for the original BeelerReuter parameters and different conductances of the SAC: (a) $g_{s}=0$, showing concordant alternans, and (b) $g_{s}=0.0065 \mathrm{~ms}^{-1}$, resulting in discordant alternans. Besides the change in $g_{s}$ all the other parameters are the same. The spacial and time discretizations are $d x=0.025 \mathrm{~cm}$, for a total length $L=6 \mathrm{~cm}$, and $d t=0.01 \mathrm{~ms}$, respectively. The pacing $T_{\text {pace }}=340 \mathrm{~ms}$ is obtained applying periodically a current of 1.0 (in nondimensional units) during $0.01 \mathrm{~ms}$ at the first 10 grid points.

Since the minimum tissue size necessary to obtain discordant alternans depends on this slope [7], we would not expect to observe discordant alternans with the modified parameters unless we consider infinitely long cables. More specifically, an estimate of the minimum tissue size to obtain alternans (for

(a)

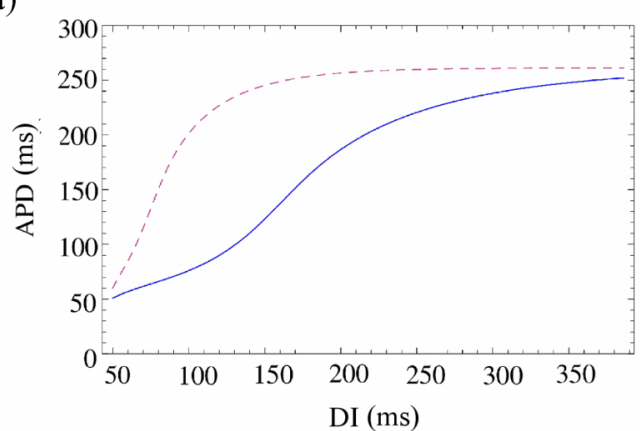

(c)

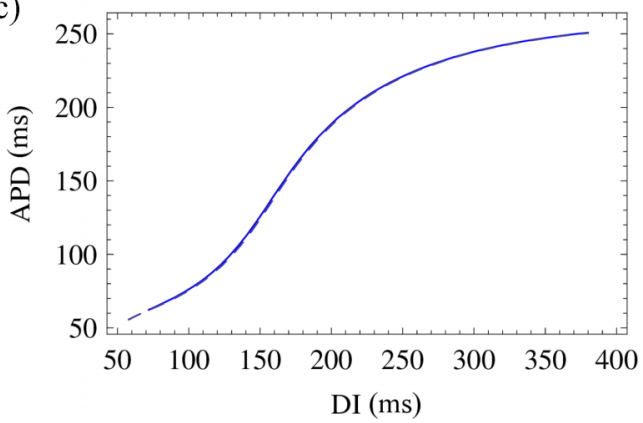

a stationary node) was given in Ref. [7] as

$$
L_{\min }=\pi \sqrt{w \Lambda} / 2
$$

with $\Lambda=c^{2} / 2(d c / d \mathrm{DI})$, and $w \sim D / c$ a coefficient related with the left-right asymmetry in diffusive coupling for a propagating pulse. For the modified parameters $d c / d \mathrm{DI}$ is very small so that $\Lambda \rightarrow \infty$ and discordant alternans never appears in tissue.

Considering now the coupled excitation-contraction system in Eqs. (1)-(4) we observe that the coupling with contraction changes the slope of the $\mathrm{CV}$ restitution curve, but not that of the APD restitution [Figs. 3(c) and 3(d)], consistent with results obtained in more realistic electromechanical models [25]. Thus, the onset of alternans (in cell) does not change, but the nature of alternans in tissue (either concordant or discordant) may change due to this coupling. From the expressions for the minimun tissue size necessary to obtain discordant alternans given in Eq. (5) the problem seems rather straightforward to solve. Just measure the slope of the $\mathrm{CV}$ restitution curve at the onset of alternans for different values of the stretch-activated current conductance $g_{s}$ and from this obtain the minimum tissue size for the transition to discordant alternans as a function of $g_{s}$. However, the situation is more complex, since the change in the slope is not constant but depends on the position in tissue at which it is measured. Thus, the formulas obtained in Ref. [7] to calculate the minimum tissue size are not valid now, and we have to obtain an equivalent expression that incorporates the effect of mechanoelectric feedback. This will be one of the goals of the following sections.

(b)

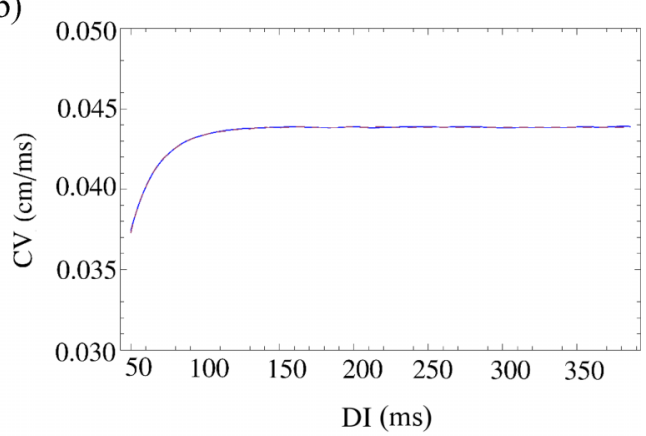

(d)

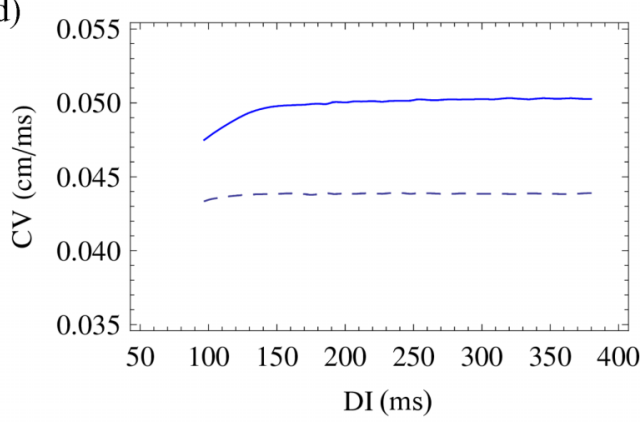

FIG. 3. (Color online) (a) APD and (b) CV restitution curves for the parameters of the Fenton-Karma model, corresponding to the original Beeler-Reuter model (dashed red lines), and the modified parameters, where we have changed the value of $\tau_{w}^{-}$from 41 to 95 ms (solid blue lines). The restitution curves are obtained in a cable of length $L=2 \mathrm{~cm}$, using a S1S2 protocol, with S1 $=450 \mathrm{~ms}$. (c) APD and (d) CV restitution curves for the modified parameters and two values of the SAC conductance, $g_{s}=0.005 \mathrm{~ms}^{-1}$ (solid line) and $g_{s}=0 \mathrm{~ms}^{-1}$ (dashed line), showing that, while the APD restitution curve remains constant, the CV restitution curve becomes steeper as $g_{s}$ is increased. 


\section{COUPLED MAPS}

The dynamics of the system can be studied by a mesoscopic description using the restitution curves to obtain coupled map equations for the APD and pacing period $T$. In general, the dynamics of the full model can be properly projected into a set of two maps (see full details in Refs. [6,7]):

$$
\begin{gathered}
A^{n+1}=f\left(T^{n}-A^{n}\right)-w \partial_{x} A^{n}+\xi^{2} \partial_{x}^{2} A^{n}, \\
T^{n}(x)=T_{\text {pace }}+\int_{0}^{x} \frac{d x^{\prime}}{c^{n}}-\int_{0}^{x} \frac{d x^{\prime}}{c^{n-1}},
\end{gathered}
$$

where $T_{\text {pace }}$ is the pacing period on one end of the cable, $T^{n}$, $c^{n}, D^{n}$, and $A^{n}$ are the stimulation interval, wave speed, DI, and APD of the $n$th pulse, and $T^{n}=A^{n}+D^{n}$. It is important to notice that Eq. (7) is strictly the definition of the period at a given position $x$ while Eq. (6) is a good approximation of the APD dynamics where the coefficients $w$ and $\xi^{2}$ are obtained from the restitution curves (see Appendix A) giving in our case $w \simeq 0.145 \mathrm{~cm}$ and $\xi^{2} \sim 0.2 \mathrm{~cm}^{2}$.

The dynamical system given by Eqs. (6) and (7) must be closed with an expression for the conduction velocity $c$. In the absence of contraction, this is just $c^{n}=c\left(D^{n}\right)$. However, contraction introduces a spatial dependence that makes the determination of $c$ nontrivial. Indeed, we have measured the $\mathrm{CV}$ along the tissue for different values of the SAC conductance parameter $g_{s}$ [Fig. 4(a)] and observed that the conduction velocity increases as the pulse travels along the

(a)

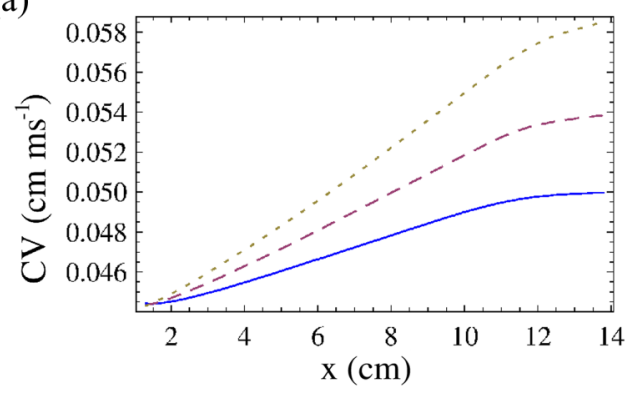

tissue, up to a point where the whole AP has entered the cable, and a constant conduction velocity is reached. Based on this observation we consider that, once the whole action potential is in the cable, the conduction velocity depends both on the diastolic interval of the previous beat and on the action potential duration of the current one, so $c^{n+1}=\operatorname{CV}\left(D^{n}, A^{n+1}\right)$. Furthermore, according to Fig. 4(b) we may assume that the dependence of conduction velocity with APD is linear. However, this general dependence is not enough to close the system since it is valid only when the full action potential has entered the cable. From Fig. 4(a) we can assume that, while the action potential is entering the cable, the conduction velocity increases linearly with the distance traveled by the pulse. This allows us to write the conduction velocity as

$$
\begin{aligned}
c^{n+1}= & \operatorname{CV}\left(D^{n}\right)+\alpha A^{n+1} S\left(x-c^{n+1} A^{n+1}\right) \\
& +\alpha x / c^{n+1}\left[1-S\left(x-c^{n+1} A^{n+1}\right)\right],
\end{aligned}
$$

where $S(\cdot)$ is a sigmoidal function that interpolates between the regions where the conduction velocity is constant or grows linearly with $x$. The coefficient $\alpha$, which effectively measures the effect of contraction on conduction velocity, can be calculated fitting the measured profiles of $\mathrm{CV}(x)$ with the function given in Eq. (8) [Fig. 4(c)], for different values of the length of the system $L$ and SAC conductance $g_{s}$. As shown in Fig. 4(e), plotting the product $\alpha L$ as a function of $g_{s}$ all the data collapses into a single curve, from which we get

$$
\alpha=\frac{g_{s}}{L}\left(0.06+2.8 g_{s}\right) .
$$

(b)

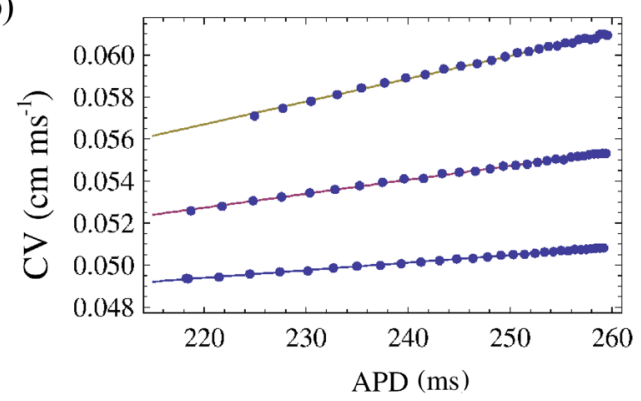

(e)

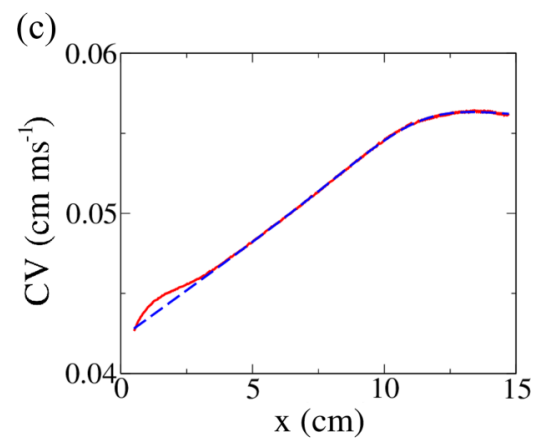

(d)
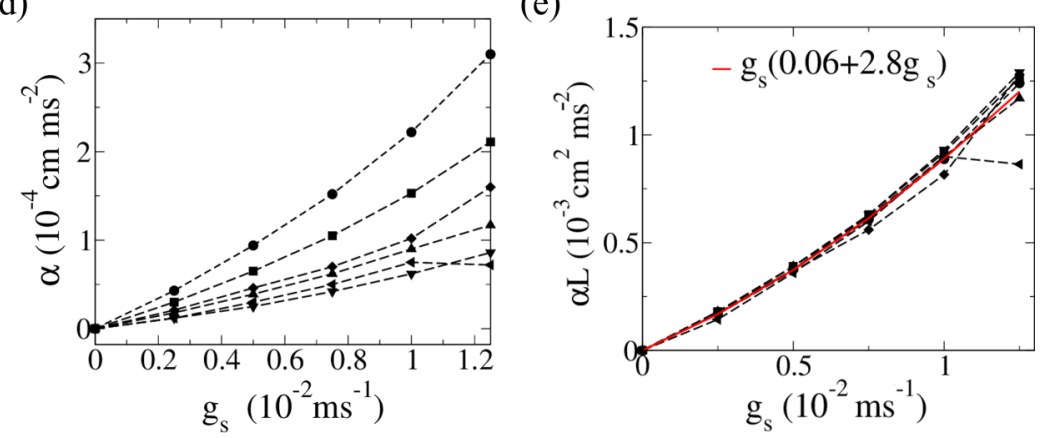

FIG. 4. (Color online) (a) Conduction velocity vs $x$ for three different values of the SAC conductance, $g_{s}=0.005 \mathrm{~ms}^{-1}$ (solid blue), $0.0075 \mathrm{~ms}^{-1}$ (long dashes, purple), and $0.01 \mathrm{~ms}^{-1}$ (short dashes, olive) for $T_{\text {pace }}=533 \mathrm{~ms}$ (dynamic) and $L=15 \mathrm{~cm}$, showing how the pulse accelerates until the whole AP is in tissue, reaching then a constant value. (b) Change of conduction velocity with APD, measured at a given point in tissue, and for several values of $g_{s}$. (c) Conduction velocity vs distance along the cable, calculated for a pacing period of $T=475 \mathrm{~ms}$ and a stretch-activated current conductance of $g_{s}=0.0125 \mathrm{~ms}^{-1}$. We fit the curve to the form given by Eq. (8), to calculate $\alpha$ for each value of $L$ and $g_{s}$. (d) Values of $\alpha$ as a function of $g_{s}$, for different lengths of the system $(L=4,6,8,10,12,15 \mathrm{~cm})$. (e) For the product $\alpha L$, all the data collapse in a single curve, giving $\alpha=g_{s}\left(0.06+2.8 g_{s}\right) / L$. 
(a)
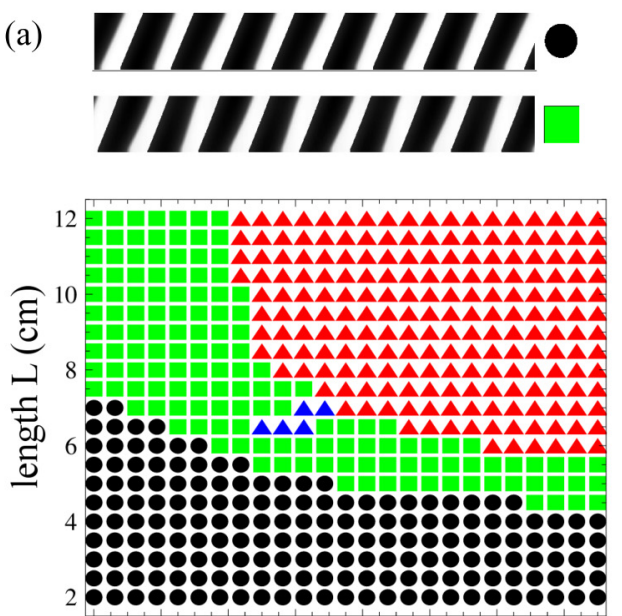

$\begin{array}{llllllll}0.000 & 0.002 & 0.004 & 0.006 & 0.008 & 0.010 & 0.012 & 0.014\end{array}$

normalized $\mathrm{g}_{\mathrm{s}}=G_{S} k_{T} / \mathrm{E}$

(c)

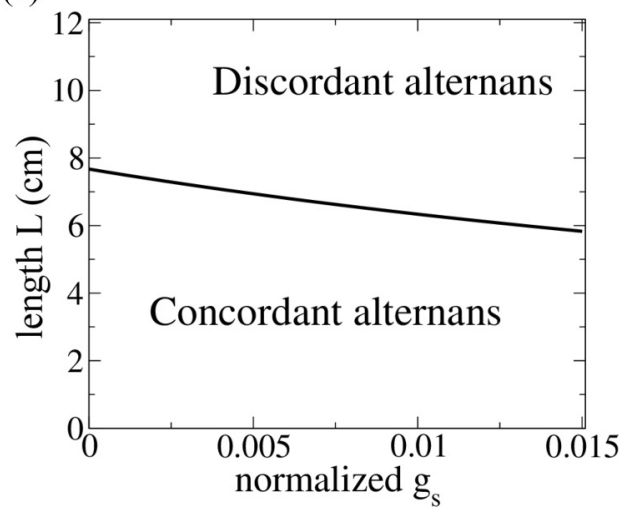

(b)
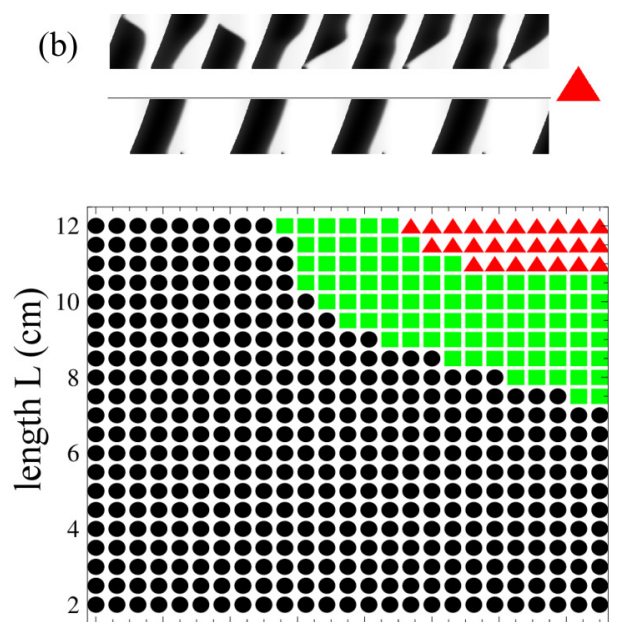

$\begin{array}{llllllll}0.000 & 0.002 & 0.004 & 0.006 & 0.008 & 0.010 & 0.012 & 0.014\end{array}$

normalized $\mathrm{g}_{\mathrm{s}}=G_{S} k_{T} / \mathrm{E}$

(d)

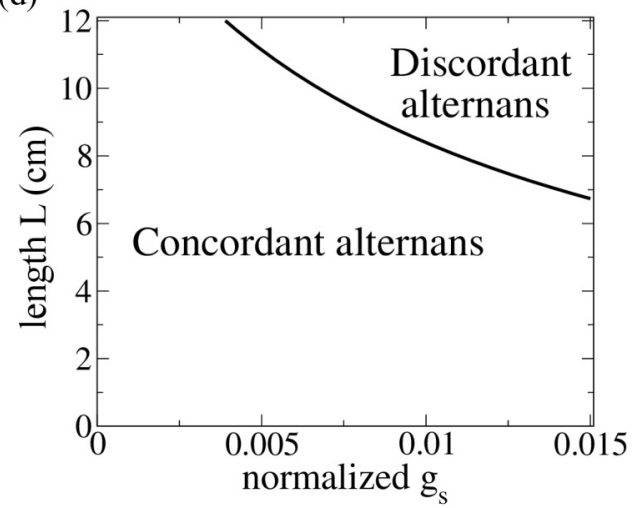

FIG. 5. (Color online) Phase plot of the different solutions obtained with Eqs. (1)-(4) as a function of the size of the system $L$ and the normalized strength of the SAC $g_{s}$, for the (a) original Beeler-Reuter parameters, and $T=343.5$ ms and (b) the modified Beeler-Reuter parameters, with $T=404 \mathrm{~ms}$. The different solutions are concordant alternans (black circles), discordant alternans (green squares), block and complex behavior (red triangles). The blue triangles correspond to suppression of alternans. Examples of the different solutions are shown above the phase diagrams, including localized and 2-1 block. In (c) and (d) we show the corresponding phase diagrams obtained with the maps (6), (7), and (8), with $S(x-c A) \equiv 0.5\{1+\tanh [1.2(x-c A) /(c A)]\}$, showing the transition from concordant to discordant alternans as a function of $g_{s}$.

With this, we close the system of equations and can compare the results of the coupled maps with the full numerical simulations of the ionic model. When doing the comparison, however, one should be cautious. Equation (8) is just an approximation: the conduction velocity actually depends on the length of the pulse at a given instant on time, and not on the duration of the pulse at a given point in space, as considered here. Furthermore, for long tissues (or fast pacing rates) where more than one pulse is present at the same time in the cable, one would have to consider the influence of this second pulse. However, we found that for typical values of $L$ and $g_{s}$, the approximation given by Eq. (8) reproduces well the numerical results from the ionic model.

The simulation of Eqs. (6), (7) together with Eqs. (8), (9), taking for the sigmoidal $S(z)=[1+\tanh (\kappa z)] / 2$, allows us to analyze the details of the transition to discordant alternans as a function of the length of the system and the strength of stretch-activated current. Both the full model (1)-(4) with the parameters of the original and modified Beeler-Reuter models and the coupled maps present a transition from concordant to discordant alternans induced by contraction (Fig. 5). For the parameters in the modified Beeler-Reuter model, the slope of $\mathrm{CV}$ restitution is negligible at the onset of alternans. Thus, discordant alternans never appears for $g_{s}=0$ [Fig. 5(b)]. Contraction alone is able to provoke the transition from concordant to discordant alternans in this case, as observed in simulations of the full model [Fig. 5(b)] and the coupled maps [Fig. 5(d)], changing $g_{s}$ for fixed $L$. For the original parameters of the Beeler-Reuter model, there is a transition to discordant alternans as the length $L$ of the cable is increased, even in the absence of contraction, due to a positive slope of the CV restitution curve at the onset of alternans. The effect of contraction is to shift this transition to lower values of $L$ [Figs. 5(a) and 5(c)].

\section{AMPLITUDE EQUATIONS}

In the previous section we showed that numerical simulations of the coupled maps are in good agreement with the results from the full model. We can, therefore, analyze in more detail the coupled maps in Eqs. (6), (7) in order to obtain 
further information about the solutions and the mechanism behind the transition from concordant to discordants alternans. Specifically, our goal is to obtain a value for the minimum length necessary to obtain discordant alternans due to the presence of stretch-activated currents.

Close to the bifurcation to alternans, we can consider small oscillations of the APD and stimulation interval, $A^{n}(x) \simeq$ $A^{*}+(-1)^{n} a(x, t), T^{n}(x) \simeq T^{*}+(-1)^{n} b(x, t)$, which are assumed to vary on slow time and spatial scales around the values at the bifurcation point. Introducing these expressions in Eqs. (6), (7), and (8) and carrying out some algebra (see Appendix B), it can be shown that the small oscillations obey a Ginzburg-Landau-type equation:

$$
\tau \partial_{t} a=\sigma a-g a^{3}-w \partial_{x} a+\xi^{2} \partial_{x}^{2} a-b .
$$

The effect of contraction is now hidden in the form of $b$, which is a complex function that depends on the slope of CV restitution and the strength of parameter $\alpha$. In the limit when there is no dispersion $(d \mathrm{CV} / d \mathrm{DI}=0)$ and $S(x-c A)$ is very steep and can be approximated by a Heaviside function, this expression greatly simplifies to (see Appendix B)

$$
b=\frac{1}{\Pi} \int_{0}^{x} \Theta\left(x^{\prime}-c^{*} A^{*}\right) a\left(x^{\prime}\right) d x^{\prime}
$$

with $\Pi=c^{* 2} / 2 \alpha$, and $c^{*}$ the conduction velocity at the onset of alternans. This is similar to the known expression due to CV restitution [6] $b \sim \int_{0}^{x} a\left(x^{\prime}\right) d x^{\prime}$ but with $b$ different from zero only for cables longer than $c^{*} A^{*}$, which corresponds to the width of the pulse at the onset of the bifurcation. The reason for this is clear: if the system size is smaller than the width of the pulses, then there is no difference in conduction velocity at alternative beats. From Eq. (8), and considering $S(\cdot)$ a Heaviside function $\Theta(\cdot)$, it is easy to see that, in that case, the $\mathrm{CV}$ does not depend on $A^{n+1}$. Beyond that point, the mechanism behind discordant alternans is the same as for conduction velocity dispersion. During alternans, a pulse with a long APD develops a higher active tension, which stretches the tissue in front of it [Fig. 6(a)]. Since the stretch-activated current appears where the tissue is distended, it is precisely in the case of long (short) APD that the tissue ahead of the front becomes more (less) depolarized. Then, a pulse with a long APD has a higher velocity than a pulse with a short APD, due to a larger stretch-activated current (Fig. 6), and catches it up as it travels along tissue, until a node is formed.

\section{A. Stability analysis}

We are interested in obtaining expressions for when the instability to alternans occurs and which is the minimum tissue size necessary to observe discordant alternans. For that, we perform a linear stability analysis of the amplitude equation (10) around the nonalternating solution $a=0$ and find an analytical expression for the eigenvalue and the eigenfunction corresponding to the marginal mode. Let us consider $a(x, t)=\exp (\Omega t / \tau) \Psi(x)$. Then, linearizing we obtain

$$
0=(\sigma-\Omega) \Psi-w \frac{d \Psi}{d x}+\xi^{2} \frac{d^{2} \Psi}{d x^{2}}-\frac{1}{\Pi} \int_{x_{0}}^{x} \Psi d x^{\prime}
$$
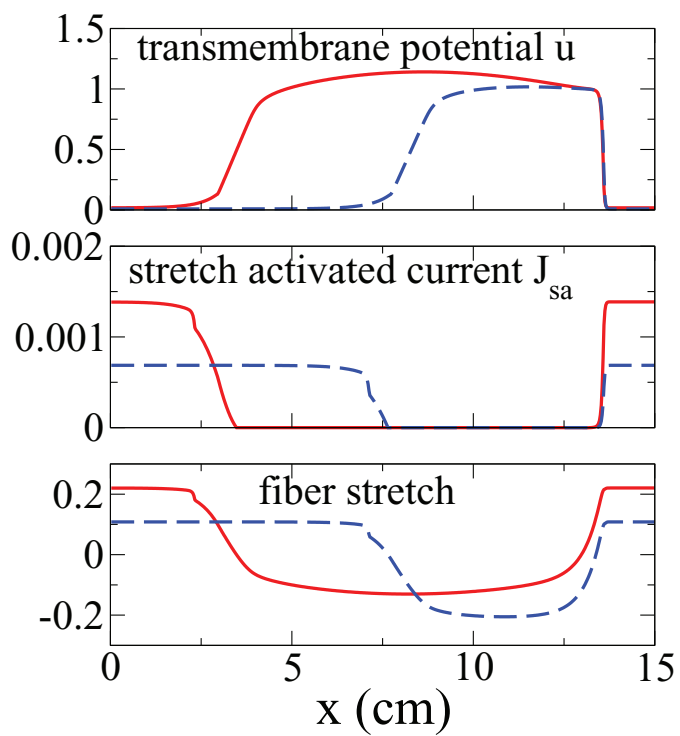

FIG. 6. (Color online) Transmembrane potential $u$, stretch activated current $J_{s a}$ and fiber stretch during alternans, corresponding to a long (solid red) and a short (dashed blue) APD, for a fiber of length $L=15 \mathrm{~cm}$, a pacing rate of $T=400 \mathrm{~ms}$, and a stretch activated current conductance $g_{s}=0.002 \mathrm{~ms}^{-1}$.

with $x_{0}=c^{*} A^{*}$. Thus, we have to solve

$$
\begin{gathered}
0=(\sigma-\Omega) \Psi_{1}-w \frac{d \Psi_{1}}{d x}+\xi^{2} \frac{d^{2} \Psi_{1}}{d x^{2}}, x<x_{0}, \\
0=(\sigma-\Omega) \Psi_{2}-w \frac{d \Psi_{2}}{d x}+\xi^{2} \frac{d^{2} \Psi_{2}}{d x^{2}}-\frac{1}{\Pi} \int_{x_{0}}^{x} \Psi_{2} d x^{\prime} x>x_{0}
\end{gathered}
$$

in a semiinfinite line $x \in[0, \infty)$, with $\Psi_{1}^{\prime}(0)=0, \Psi_{1}\left(x_{0}\right)=$ $\Psi_{2}\left(x_{0}\right)$, and $\Psi_{1}^{\prime}\left(x_{0}\right)=\Psi_{2}^{\prime}\left(x_{0}\right)$.

Let us introduce $\tilde{\Omega}=\Omega-\sigma$ and look first for a solution of $\Psi_{1}$ in the form of exponential modes $\Psi_{1} \sim e^{\lambda x}$. We get the eigenvalue equation

$$
0=-\tilde{\Omega}-w \lambda+\xi^{2} \lambda^{2}
$$

so

$$
\lambda=\frac{1}{2 \xi^{2}}\left[w \pm \sqrt{w^{2}+4 \tilde{\Omega} \xi^{2}}\right]
$$

and the general solution as

$$
\Psi_{1}=\left[A e^{\lambda_{1} x}+B e^{\lambda_{2} x}\right],
$$

being $\quad \lambda_{1}=\left[w+\sqrt{w^{2}+4 \tilde{\Omega} \xi^{2}}\right] / 2 \xi^{2} \equiv\left(w / 2 \xi^{2}\right)[1+$ $\sqrt{1+\phi}]$ and $\lambda_{2}=\left[w-\sqrt{w^{2}+4 \tilde{\Omega} \xi^{2}}\right] / 2 \xi^{2} \equiv\left(w / 2 \xi^{2}\right)[1-$ $\sqrt{1+\phi}]$, with $\phi=4 \tilde{\Omega} \xi^{2} / w^{2}$.

We can now write the condition of zero derivative at $x=0$ as

$$
\left.\frac{d \Psi_{1}}{d x}\right|_{x=0}=A \lambda_{1}+B \lambda_{2}=0
$$


which makes

$$
\begin{aligned}
\Psi_{1}(x)= & C_{1} w / \xi^{2} e^{w x / 2 \xi^{2}}\left[\sinh \frac{w x \sqrt{1+\phi}}{2 \xi^{2}}\right. \\
& \left.-\sqrt{1+\phi} \cosh \frac{w x \sqrt{1+\phi}}{2 \xi^{2}}\right] .
\end{aligned}
$$

Thus, at $x=x_{0}, \Psi_{1}$ typically diverges exponentially, as $\Psi_{1} \sim e^{w x_{0} / \xi^{2}}$ unless $\phi \sim e^{-w x_{0} / \xi^{2}}$ in which case we can obtain modes that are order one in the whole domain. In this case we can set $\phi=\tilde{\phi} e^{-w x_{0} / \xi^{2}}$, with $\tilde{\phi} \sim O(1)$. After some algebra, for $\Psi_{1}(x)$ we get the solution at first order:

$\Psi_{1}(x) \simeq \frac{w}{\xi^{2}} C_{1}\left[-1+\frac{1}{4}\left(\frac{w x}{\xi^{2}}-1\right) \tilde{\phi} e^{-w x_{0} / \xi^{2}}-\frac{\tilde{\phi}}{4} e^{w\left(x-x_{0}\right) / \xi^{2}}\right]$.

In order to match $\Psi_{2}$ and $\Psi_{1}$ at $x=x_{o}$ we can use the fraction $\Psi_{1 f}=\Psi_{1}^{\prime} / \Psi_{1}$ evaluated at $x=x_{o}$, which is independent of $C_{1}$. Then,

$$
\begin{aligned}
\Psi_{1 f}\left(x=x_{0}\right) & =\frac{\lambda_{1} \lambda_{2}\left(e^{\lambda_{1} x_{0}}-e^{\lambda_{2} x_{0}}\right)}{\lambda_{2} e^{\lambda_{1} x_{0}}-\lambda_{1} e^{\lambda_{2} x_{0}}} \\
& =-\frac{w \phi}{2 \xi^{2}} \frac{\tanh \frac{w x_{0} \sqrt{1+\phi}}{2 \xi^{2}}}{\tanh \frac{w x_{0} \sqrt{1+\phi}}{2 \xi^{2}}-\sqrt{1+\phi}},
\end{aligned}
$$

which after imposing $\phi=\tilde{\phi} e^{-w x_{0} / \xi^{2}}$ reads $\Psi_{1 f}\left(x=x_{0}\right) \simeq$ $\left(w / \xi^{2}\right) \tilde{\phi} /(4+\tilde{\phi})$.

Now we have to solve the equation for $\Psi_{2}$ matching to the slope given by (21) at $x=x_{0}$. A general solution can be searched in the form $\Psi_{2}=A e^{q\left(x-x_{1}\right)}$ where $q$ is a complex number. Introducing this function in Eq. (14) one notices that using the complex conjugate of $q$ we can eliminate the new term appearing in the integral. We find thus a particular solution which reads:

$$
\Psi_{2}=C_{2}\left[e^{q\left(x-x_{1}\right)}+e^{q^{*}\left(x-x_{1}\right)}\right]=C_{2} e^{-\rho\left(x-x_{1}\right)} \cos \left[k\left(x-x_{1}\right)\right],
$$

where $q^{*}$ is the complex conjugate of $q=-\rho+i k$ with $\rho$ and $k$ being real values. Notice that the scale of $L_{\min }$ is given by the first zero of this function, which is directly related to the

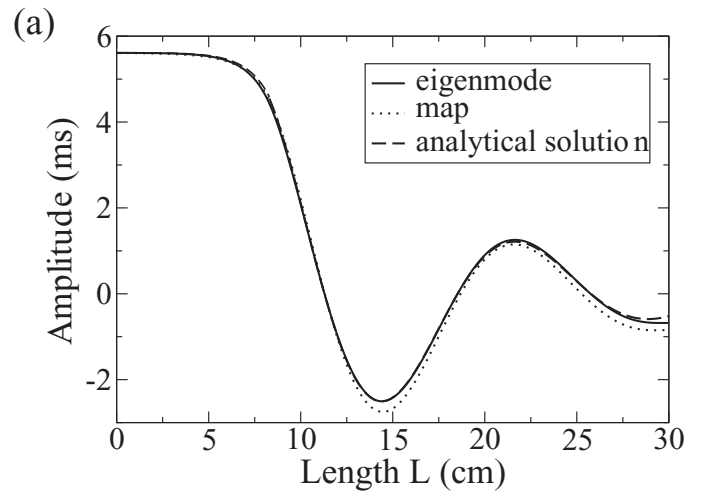

wave number $k$. Imposing that Eq. (22) satisfies the amplitude equation we get, after some algebra, the conditions

$$
\begin{gathered}
\tan k\left(x_{0}-x_{1}\right)=\frac{\rho}{k}, \\
\tilde{\Omega}+\xi^{2} k^{2}=3 \xi^{2} \rho^{2}+2 w \rho, \\
w+2 \xi^{2} \rho=\frac{1}{\Pi\left(k^{2}+\rho^{2}\right)} .
\end{gathered}
$$

Besides this, we have to impose the matching condition at $x=x_{0}, \Psi_{1 f}=\Psi_{2 f}$, that results in

$$
-2 \rho=\frac{\lambda_{1} \lambda_{2}\left(e^{\lambda_{1} x_{0}}-e^{\lambda_{2} x_{0}}\right)}{\lambda_{2} e^{\lambda_{1} x_{0}}-\lambda_{1} e^{\lambda_{2} x_{0}}} \simeq \frac{w}{\xi^{2}} \frac{\tilde{\phi}}{4+\tilde{\phi}}=\frac{w}{\xi^{2}} \frac{\tilde{\Omega}}{w^{2} / \xi^{2}+\tilde{\Omega}} .
$$

The four equations (23)-(26) determine the four unknowns of the problem, i.e., $\tilde{\Omega}, k, \rho$, and $x_{1}$.

To check the validity of the previous solution, we solved numerically the linear eigenvalue problem associated with (10), linearized and discretized in space, using a finite difference representation of the derivatives and the trapezoidal rule for the integral. Looking for exponentially growing or decaying solutions $a_{i}(t) \sim a_{i} e^{\Omega t / \tau}$, we obtain a set of $N$ coupled linear algebraic equations,

$$
\begin{aligned}
\Omega a_{i}= & \sigma a_{i}-\frac{w}{2 d x}\left(a_{i+1}-a_{i-1}\right)+\frac{\xi^{2}}{d x^{2}}\left(a_{i+1}+a_{i-1}-2 a_{i}\right) \\
& -\frac{d x}{\Lambda} \sum_{j=j_{0}}^{i-1} \frac{1}{2}\left(a_{j}+a_{j+1}\right), \quad i=1, \ldots, N
\end{aligned}
$$

where $L=N d x, j_{0}=x_{0} / d x$, with $x_{0}=c^{*} A^{*}$, and $d x=0.05$ $\mathrm{cm}$. The nonflux boundary conditions now become $a_{0}=a_{2}$, and $a_{N+1}=a_{N-1}$. The resulting eigenvalue problem is then solved for the complex growth rate $\Omega=\Omega_{r}+i \Omega_{i}$, and the corresponding eigenmodes.

In Fig. 7 we show a comparison of the eigenmodes obtained from the analytical solution, the solution of the eigenvalue problem and the full maps, showing an excellent agreement. This is specially remarkable for the comparison with the full maps and stresses the validity of the amplitude equation's formalism.

(b)

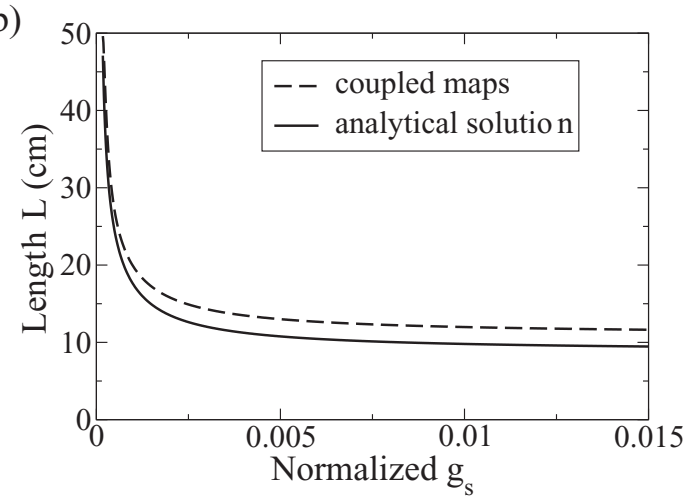

FIG. 7. (a) Comparison of the solutions given by the full maps (dotted line), with $S(z) \equiv \Theta(z)$, the solutions of the eigenvalue problem (solid line), and the analytical solution (dashed line). (b) Onset of discordant alternans, as given by Eq. (32) (solid line) and the full maps (dashed line), with $S(z) \equiv \Theta(z)$, and $S\left(z^{n}\right) \equiv 0.5\left\{1+\tanh \left[1.2\left(x-c^{n} A^{n}\right) / c^{n} A^{n}\right]\right\}$. 


\section{B. Evaluation of $L_{\min }$ as a function of the SAC conductance $g_{s}$}

Once we have the analytical solution, we can compute the minimum size of tissue necessary to obtain alternans $\left(L_{\min }\right)$ as the first zero of the general solution $\Psi$. In this case, this is the first zero of $\Psi_{2}$, given by

$$
L_{\text {min }}=x_{1}+\frac{\pi}{2 k}=x_{0}+\frac{1}{k}\left[\frac{\pi}{2}-\arctan \frac{\rho}{k}\right],
$$

where $k$ should be determined from Eqs. (24)-(26). When $\phi=$ $\tilde{\phi} e^{-w x_{0} / 2 \xi^{2}}$, we can neglect the term $\tilde{\Omega}$ in Eq. (24), so we get

$$
\begin{gathered}
k^{2}=3 \rho^{2}+\frac{2 w}{\xi^{2}} \rho, \\
\rho\left(\rho+w / 2 \xi^{2}\right)^{2}=\frac{1}{8 \xi^{2} \Pi} .
\end{gathered}
$$

Then, for a given value of $\Pi$, Eq. (30) gives the value of $\rho$ that then can be used to obtain $k$ and $\tilde{\Omega}$ from Eqs. (29) and (26). In the limit $\Pi \rightarrow \infty$ we have two possible solutions to Eq. (30): $\rho=0$, or $\rho=-w / 2 \xi^{2}$. However, for large values of $\Pi$ looking for a solution around $\rho=0$ gives a very bad approximation. For both typical and large values of $\Pi, \rho$ is indeed small but not extremely close to zero. In this circumstance one can neglect the cubic term in Eq. (30) and obtain a very good approximation solving the quadratic equation. One obtains

$$
\rho=\frac{w}{8 \xi^{2}}\left[\sqrt{1+\frac{8 \xi^{4}}{w^{3} \Pi}}-1\right],
$$

which introduced in Eq. (29) and (28) produces

$$
L_{\min } \simeq x_{0}+\sqrt{w \Pi}\left[\frac{\pi}{2}-\arctan \frac{\xi^{2}}{2 \sqrt{w^{3} \Pi}}\right]
$$

with $x_{0}=c^{*} A^{*}$. Recalling that $\Pi=c^{2} / 2 \alpha$ and substituting the expression for $\alpha=g_{s}\left(0.06+2.8 g_{s}\right) / L$ obtained in Fig. 4 , one obtains the limits $L_{\min } \sim 1 / g_{s}$ for $g_{s} \rightarrow 0$ and $L_{\text {min }} \rightarrow c^{*} A^{*}$, when $g_{s} \gg 1$ (see Fig. 5), consistent with the results obtained with coupled maps and the full ionic model.

\section{CONCLUSION}

In this paper, we have considered a mechanoelectric model that couples active tension directly to a simplified model of the cardiac excitability and analyzed an equivalent coupled map scenario, to show that tissue contractions have a proarrythmic effect and may cause discordant alternans. The model shows that a small contraction effect suffices to induce the transition from concordant to dicordant alternans via its influence on the conduction velocity properties. In our model, stretch increases the conduction velocity, in accordance to what has been observed in other numerical studies under similar conditions [25]. The reason is that stretch opens the stretch activated channels, elevating the resting potential, thus making it easier to depolarize.

We have performed a detailed analytical treatment in terms of coupled maps and their amplitude equation. This has allowed us to uncover the basic mechanism for discordant alternans. As an effect of the stretch-activated current, a pulse with a long APD has a higher velocity than a pulse with a short
APD, so the two pulses approach, decreasing the DI and thus the APD of the long pulse, until a node is formed. This happens in situations where the derivative of the conduction velocity with the diastolic interval is zero, which, in the absence of stretch-activated currents, would make discordant alternans impossible. An analysis of the amplitude equation has allowed us to show that the minimum length of the system needed to observe discordant alternans increases as the inverse of the strength of the stretch-activated as this goes to zero.

A limitation of our formulation is that it considers linear elasticity, whereas the elastic response of real tissue is known to be highly nonlinear. Still, given that the transition from concordant to discordant alternans occurs even at very small values of the stretch-activated conductance, this would not affect the qualitative prediction of the model. Perhaps a bigger uncertainty remains in the correct form of the stretch activated current. In fact, it has been observed that the conductance of this current is not a constant, but varies with time, having an activation time of $\sim 20 \mathrm{~ms}$ to peak current, and then a decay to half the peak current value in about $50 \mathrm{~ms}$ [26]. The simple formulation of the SAC used in this paper would consider average values and assume that the transients are fast in the time scale of development of active tension. Furthermore, the coupling between excitation and contraction in our model is very simplistic and does not take into account the role played by intracellular calcium. In this respect, it cannot account for alternans due to intracellular calcium, or for changes in the resting potential due to the effect of stretch on the dynamics of calcium handling [27].

More importantly, our analysis assumes an increase of conduction velocity with stretch. Indeed, there exists experimental and numerical evidence showing that this is the case for moderate values of stretch [25,28], although the relation has often been observed to be biphasic [28]. At larger values of stretch the increase in the resting potential inactivates the sodium channels, resulting in slower conduction and even conduction block. However, there are also experiments where conduction velocity has been observed to be constant or to decrease with stretch [28-31]. Thus, the validity of our assumptions is not clear in this point. Even so, under the conditions considered in the paper, the scenario unveiled here is general and the conclusions hold generally. Whenever stretch is created in front of the pulse increasing the conduction velocity, a small stretch-activated current together with the nonlocal nature of the interaction produce discordant alternans.

An open question is the generalization of our results to two- (2D) or three-dimensional tissue, which would be the relevant case for the heart. In a $2 \mathrm{D}$ tissue, in the case where radial symmetry can be assumed, the effect of stretch is the same as in 1D tissue [32] and one would expect similar results. However, in two dimensions the effect of contraction is very dependent on the position of the pacing point and the form (and type) of the boundaries [33,34]. This is specially relevant given the complex interaction between walls in a real heart, a question that cannot be addressed without further knowledge of heart contraction. Also interesting is the effect of MEF in the case where a spiral is present in 2D tissue with standard boundary conditions. MEF is known to produce a drift of the spiral, proportional to the strength of the SAC [25]. However, in the presence of alternans, the topology of the spiral induces 
the formation of nodal lines [35], which can present complex dynamics [36,37]. Our results in 1D suggest that one would expect a transition from a straight nodal line (corresponding to concordant alternans in the radial direction) to a spiraling one (corresponding to discordant alternans) due to MEF, at least as long as there are not multiple arms of the spiral in the tissue. Analyzing this latter case is a natural next step to understand the effect of MEF in cardiac alternans.

\section{ACKNOWLEDGMENTS}

M.R. and M.B. acknowledge financial support from the Deutsche Forschungsgemeinschaft (DFG) through GRK1558 and from the Deutscher Akademischer Austauschdienst (DAAD) through the Acciones Integradas program. E.A.L. and B.E. acknowledge financial support from MICINN through coordinated project FIS2011-28820-C02-01 and through the Acciones Integradas program (DE2009-0035).

\section{APPENDIX A: CALCULATION OF THE COEFFICIENTS $w, \xi^{2}$}

We will calculate $w$ and $\xi^{2}$ as sketched in Ref. [7]. For that, we have to consider parameters for which there is discordant

(a)

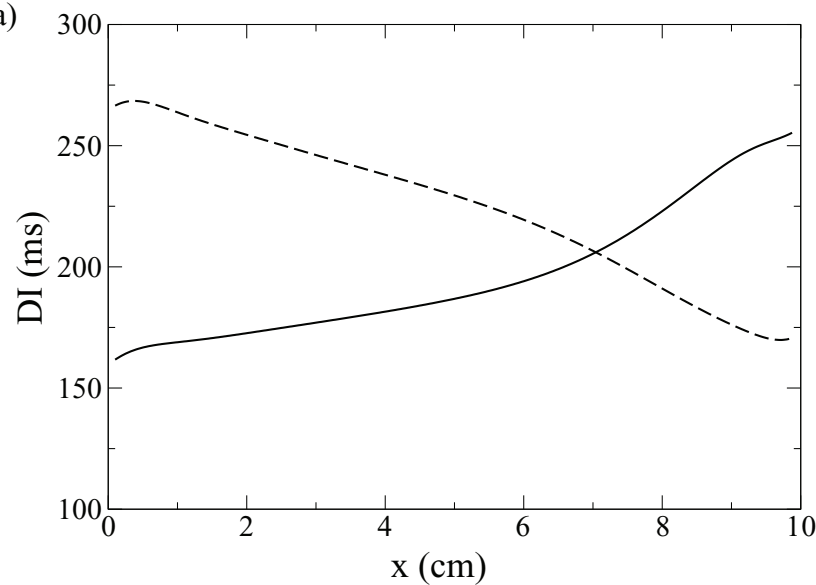

(c)

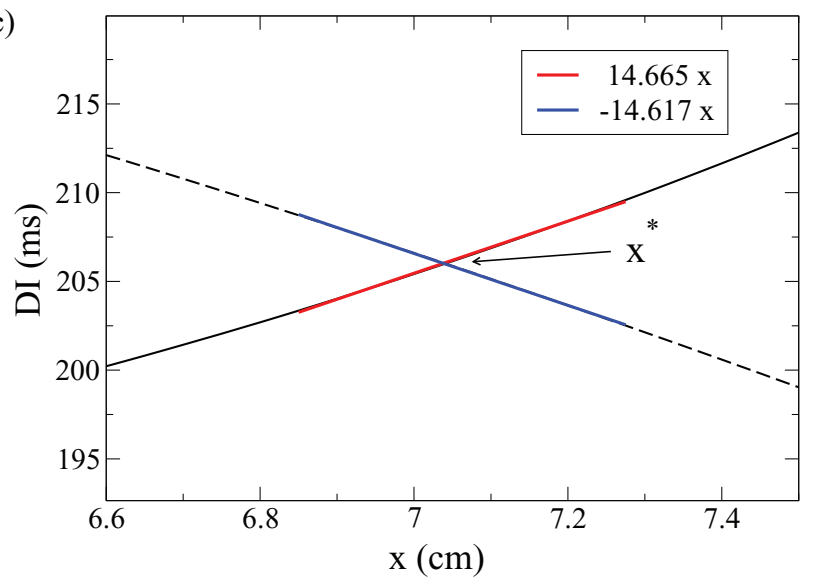

alternans in the tissue. Computing $D(x)$, we can find the position of a node $x^{*}$, for which $D^{n+1}\left(x^{*}\right)=D^{n}\left(x^{*}\right)=D^{*}$. Since, from the restitution curve $A=f(D)$, we would expect to see the same value of the APD at two consecutive beats at that value of DI. If there is a difference, it must be due to the spatial terms, that cause a splitting of the restitution curves:

$$
A^{n+1}(x)=f_{S 1 S 2}\left[D^{n}(x)\right]-w \partial_{x} D^{n}(x)+\xi^{2} \partial_{x}^{2} D^{n}(x) .
$$

Thus, since close to the node in DI, the second derivative is almost zero:

$$
A^{n+1}\left(x^{*}\right)-A^{n}\left(x^{*}\right) \simeq w\left[\partial_{x} D^{n-1}\left(x^{*}\right)-\partial_{x} D^{n}\left(x^{*}\right)\right] .
$$

Then

$$
w \simeq \frac{A^{n+1}\left(x^{*}\right)-A^{n}\left(x^{*}\right)}{\partial_{x} D^{n-1}\left(x^{*}\right)-\partial_{x} D^{n}\left(x^{*}\right)} .
$$

In the example shown in Fig. 8, we have

$$
w \simeq \frac{4.25 \mathrm{~ms}}{29.28 \mathrm{~ms} / \mathrm{cm}}=0.145 \mathrm{~cm}
$$

For the coefficient $\xi^{2}$ we will take the estimate $\xi^{2} \sim D \times$ $\mathrm{APD} \sim 0.2 \mathrm{~cm}^{2}$.

(b)

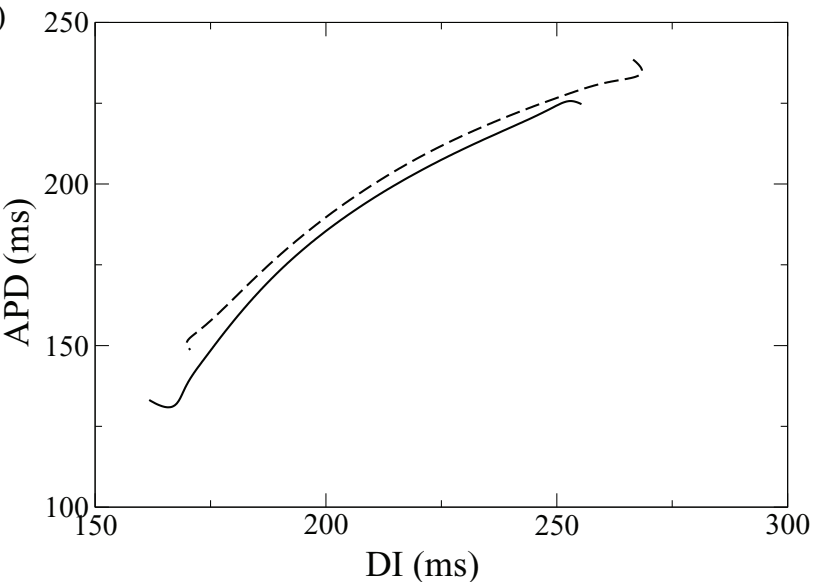

(d)

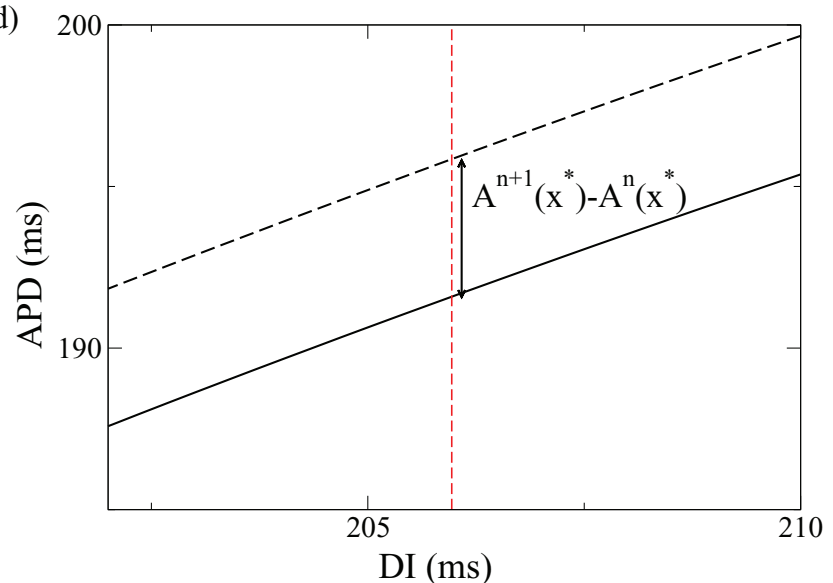

FIG. 8. (Color online) (a) Spatial distribution of DI at two consecutive beats during discordant alternans and (b) APD vs DI for those two beats. In (c) and (d) we show the same graphs closer to the node where $D^{n+1}\left(x^{*}\right)=D^{n}\left(x^{*}\right)$. From these we calculate the gradient in DI $(x)$ at two consecutive beats and the splitting of the restitution curves. Parameters: $T=400 \mathrm{~ms}, L=10 \mathrm{~cm}, g=1.45 \times 10^{-3}$. 


\section{APPENDIX B: DERIVATION OF THE AMPLITUDE EQUATIONS}

\section{General approach}

To derive the amplitude equation (10) from the maps (6), (7), we first consider the expression for the pacing period at a given point in tissue, which reads:

$$
\begin{aligned}
T^{n}(x)= & \int_{0}^{L} \frac{d x^{\prime}}{c\left[D^{n}\left(x^{\prime}\right), A^{n+1}\left(x^{\prime}\right), A^{n}\left(x^{\prime}\right)\right]} \\
& -\int_{0}^{L} \frac{d x^{\prime}}{c\left[D^{n-1}\left(x^{\prime}\right), A^{n}\left(x^{\prime}\right), A^{n-1}\left(x^{\prime}\right)\right]},
\end{aligned}
$$

or, differentiating,

$$
\begin{aligned}
\frac{d T^{n}(x)}{d x}= & \frac{1}{c\left[D^{n}(x), A^{n+1}(x), A^{n}(x)\right]} \\
& -\frac{1}{c\left[D^{n-1}(x), A^{n}(x), A^{n-1}(x)\right]}
\end{aligned}
$$

As explained in the text, we will assume that the dependence of $\mathrm{CV}$ on the current and previous APDs is negligible so that combining Eqs. (B2) and (8) we can write

$$
\begin{aligned}
\frac{d T^{n}}{d x}= & \frac{1}{c\left(D^{n}\right)+\alpha A^{n+1} S\left(z_{n+1}\right)+\alpha x / c^{n+1}\left[1-S\left(z_{n+1}\right)\right]} \\
& -\frac{1}{c\left(D^{n-1}\right)+\alpha A^{n} S\left(z_{n}\right)+\alpha x / c^{n}\left[1-S\left(z_{n}\right)\right]},
\end{aligned}
$$

where we recall here again that $z_{n}=x-c^{n} A^{n}$.

Now, if oscillations are small because we are close to the onset of alternans, we can expand the period and the APD around their value at the onset $\left(T^{*}, A^{*}\right)$ :

$$
\begin{aligned}
& T^{n}(x) \simeq T^{*}+(-1)^{n} b(x, t), \\
& A^{n}(x) \simeq A^{*}+(-1)^{n} a(x, t),
\end{aligned}
$$

where $a / A^{*}, b / T^{*} \ll 1$ vary slowly in time and space. The general goal before obtaining the final amplitude equation is to be able to write $d b / d x$ as a function of $b$ and $a$. To do it, we expand the previous expressions up to first order in $\alpha$ and $a, b$ to get

$$
c\left(D^{n}\right) \simeq c\left(D^{*}\right)+c^{*}(-1)^{n}(b-a) \equiv c^{*}+c^{*}(-1)^{n}(b-a),
$$

$$
\begin{aligned}
S\left(z_{n}\right) \simeq & S\left(x-\left[c^{*}+c^{*}(-1)^{n}(b-a)\right]\left[A^{*}+(-1)^{n} a\right]\right) \\
\simeq & S\left(x-c^{*} A^{*}\right)-S^{\prime}\left(x-c^{*} A^{*}\right)(-1)^{n} \\
& \times\left[c^{*} a+c^{*} A^{*}(b-a)\right] \\
\equiv & S\left(z^{*}\right)-S^{\prime}\left(z^{*}\right)(-1)^{n} G(b, a),
\end{aligned}
$$

where, in Eq. (B7), we have used that, at constant pacing, $T^{n}=A^{n}+D^{n}$. Notice that to shorten the notation we have defined $c^{*} \equiv c\left(D^{*}\right), c^{*} \equiv d c / d D\left(D^{*}\right), S\left(z^{*}\right) \equiv S(x-$ $\left.c^{*} A^{*}\right), S^{\prime}\left(z^{*}\right) \equiv S^{\prime}\left(x-c^{*} A^{*}\right)$, and $G(b, a)=\left(c^{*}-c^{*} A^{*}\right) a+$ $c^{*} A^{*} b$. We can now use this expansion to expand the denominators of Eq. (B4) to get

$$
\begin{aligned}
c\left(D^{n}\right) & +\alpha A^{n+1} S+\alpha x / c^{n+1}(1-S) \\
\simeq & c^{*}+c^{\prime *}(-1)^{n}(b-a)+\alpha\left[A^{*}+(-1)^{n+1} a\right] \\
& \times\left[S\left(z^{*}\right)+S^{\prime}\left(z^{*}\right)(-1)^{n+1} G(b, a)\right] \\
+ & \alpha x / c^{*}\left[1-S\left(z^{*}\right)-S^{\prime}\left(z^{*}\right)(-1)^{n+1} G(b, a)\right] \\
& \times\left[1-(-1)^{n} \frac{c^{*}}{c^{*}}(b-a)\right] \\
\simeq & c^{*}+c^{\prime *}(-1)^{n}(b-a)-\alpha \frac{x c^{*}}{c^{* 2}}(-1)^{n}(b-a)+(-1)^{n+1} \\
& \times \alpha S^{\prime}\left(z^{*}\right) c^{\prime *} A^{*}\left(A^{*}-x / c^{*}\right) b \\
+ & \alpha(-1)^{n+1} a\left[S\left(z^{*}\right)+S^{\prime}\left(z^{*}\right)\left(c^{*} A^{*}-c^{*} A^{* 2}-x\right.\right. \\
+ & \left.\left.c^{*} A^{*} x / c^{*}\right)\right] \\
+ & \alpha\left[S\left(z^{*}\right) A^{*}+\left[1-S\left(z^{*}\right)\right] x / c^{*}\right] .
\end{aligned}
$$

The term $\alpha\left[S\left(z^{*}\right) A^{*}+\left(1-S\left(z^{*}\right)\right) x / c^{*}\right]$ is just the change in conduction velocity due to the APD that is present even in the absence of alternans [see Fig. 3(c) in the main text]. The last term in Eq. (B9) is the combined effect due to contraction and alternans and has two contributions: $\alpha a S\left(z^{*}\right)$, which takes into account the fact that the APD is changing in consecutive beats, and so does its effect on the velocity, and $\alpha a S\left(z^{* *}\right)\left(A^{*} c^{*}-x\right)$, which takes into account that the length of the AP is changing from beat to beat, and so does the point at which the speed reaches a plateau.

Using the former expression, it is not difficult to expand

$$
\begin{aligned}
\frac{1}{c^{n}}-\frac{1}{c^{n-1}} \simeq & -(-1)^{n} \frac{2}{c^{* 2}}\left\{c^{\prime *}(b-a)+\alpha x c^{*} / c^{* 2}(b-a)\right. \\
& -\alpha S^{\prime}\left(z^{*}\right) c^{*} A^{*}\left(A^{*}-x / c^{*}\right) b-\alpha a\left[S\left(z^{*}\right)\right. \\
& \left.\left.+S^{\prime}\left(z^{*}\right)\left(c^{*} A^{*}-c^{\prime *} A^{* 2}-x+c^{*} A^{*} x / c^{*}\right)\right]\right\}
\end{aligned}
$$

in order to compute $d T^{n} / d x$ and, in this way, to obtain an expression for $b$, since $d T^{n} / d x \simeq(-1)^{n} d b / d x$, using that oscillations in pacing period are much smaller that oscillations in APD, i.e., $b \ll a$ :

$$
\begin{aligned}
\frac{d b}{d x}= & \frac{1}{\Lambda}\left(1+\frac{x}{2 \Pi}\right) a+\frac{1}{\Pi} a\left[S\left(z^{*}\right)\right. \\
& \left.+S^{\prime}\left(z^{*}\right)\left(c^{*} A^{*}-c^{*} A^{* 2}-x+c^{\prime *} A^{*} x / c^{*}\right)\right],
\end{aligned}
$$

where we have defined the length scales $\Lambda=c^{* 2} / 2 c^{* *}$ and $\Pi=c^{* 2} / 2 \alpha$, which measure the relative importance of $\mathrm{CV}$ dispersion and excitation-contraction coupling, respectively.

\section{Amplitude equation and its simplification}

Once Eq. (B11) has been obtained, we can now use the results (and derivation) of the amplitude equation as described in Ref. [7]. Taking into account that the map for the APD, Eq. (6), is not modified, one obtains

$$
\tau \partial_{t} a=\sigma a-g a^{3}-w \partial_{x} a+\xi^{2} \partial_{x}^{2} a-b
$$


with $b$ obtained integrating Eq. (B11), finally resulting in the amplitude equation

$$
\begin{aligned}
\tau \partial_{t} a= & \sigma a-g a^{3}-w \partial_{x} a+\xi^{2} \partial_{x}^{2} a \\
& -\int_{0}^{x}\left\{\frac{1}{\Lambda}\left[1+\frac{x}{2 \Pi}\right]\right. \\
& \left.+\frac{1}{\Pi}\left[S\left(z^{\prime *}\right)+S^{\prime}\left(z^{*}\right)\left(c^{*} A^{*}-x^{\prime}\right)\left(1-\frac{c^{*} A^{*}}{\Lambda}\right)\right]\right\} a d x^{\prime}
\end{aligned}
$$

Solving the above equation exactly is cumbersome, but we can obtain a fairly simplified expression by doing some approximations. First, let us assume that, without contraction, dispersion is negligible. This is actually the case for the second set of parameters, which were chosen precisely to make $\Lambda \rightarrow \infty$. The amplitude equation for $a$ reads as

$$
\begin{aligned}
\tau \partial_{t} a= & \sigma a-g a^{3}-w \partial_{x} a+\xi^{2} \partial_{x}^{2} a \\
& -\int_{0}^{x}\left\{\frac{1}{\Pi}\left[S\left(z^{*}\right)+S^{\prime}\left(z^{*}\right)\left(c^{*} A^{*}-x^{\prime}\right)\right]\right\} a d x^{\prime}
\end{aligned}
$$

Given that $S(z)$ is a rather steep function let us write the hyperbolic tangent as

$$
S(z)=\Theta(z)+R(z), \quad S^{\prime}(z)=\frac{\kappa}{2 \cosh ^{2}(\kappa z)}
$$

with $R(z)$ being the difference between the $\Theta$ function and $S(z)$. We can then write the amplitude equations as

$$
\begin{aligned}
\tau \partial_{t} a= & \sigma a-g a^{3}-w \partial_{x} a+\xi^{2} \partial_{x}^{2} a \\
& -\frac{1}{\Pi} \int_{0}^{x} \Theta\left(x^{\prime}-c^{*} A^{*}\right) a d x^{\prime} \\
& -\frac{1}{\Pi} \int_{0}^{x}\left[R\left(x^{\prime}-c^{*} A^{*}\right)-\frac{\kappa\left(x^{\prime}-c^{*} A^{*}\right)}{2 \cosh ^{2} \kappa\left(x^{\prime}-c^{*} A^{*}\right)}\right] a d x^{\prime} .
\end{aligned}
$$

Now we can consider a rather important approximation. For large $\kappa$ values in $S(z)$, the difference between the functions $S(z)$ and $\Theta(z)$ becomes is small, and we can take

$$
\begin{aligned}
& \int_{0}^{x} \Theta\left(x^{\prime}-c^{*} A^{*}\right) a d x^{\prime}=\int_{c^{*} A^{*}}^{x} a d x^{\prime}, \\
& \int_{0}^{x} R\left(x^{\prime}-c^{*} A^{*}\right) a d x \simeq 0, \\
& \int_{0}^{x} S^{\prime}\left(x^{\prime}-c^{*} A^{*}\right)\left(x^{\prime}-c^{*} A^{*}\right) a d x^{\prime} \\
& \simeq \int_{0}^{x}\left(x^{\prime}-c^{*} A^{*}\right) \delta\left(x^{\prime}-c^{*} A^{*}\right) a d x=0,
\end{aligned}
$$

getting

$$
\tau \partial_{t} a=\sigma a-g a^{3}-w \partial_{x} a+\xi^{2} \partial_{x}^{2} a-\frac{1}{\Pi} \int_{c^{*} A^{*}}^{x} a d x^{\prime} .
$$

This amplitude equation is a good approximation of the coupled map dynamics whenever the amplitude of the alternation is small and when the $\Theta$ function is a good representation of the sigmoidal function $S(z)$. The first is accomplished if we are close to the alternans threshold; the second can depend on the particular implementation of the sigmoid function. We have used $S=\tanh (x-c A)$ so far, but other implementations like $S=\tanh [(x-c A) / c A]$ (which leads to the same amplitude equation) are possible. In the particular case of $S=\tanh [(x-c A) / c A]$, the amplitude equation is a better approximation of the coupled model for shorter lengths and larger mechanoelectric coupling even when one could expect the amplitude equation to fail. Let us note that, using simulations of the full coupled model, we have tested these approximation. We know that they generally overestimate the value of the characteristic length scale needed to obtain discordant alternans but give the right order of magnitude.
[1] P. J. Schwartz and A. Malliani, Am. Heart J. 89, 45 (1975); J. M. Smith, E. A. Clancy, R. Valeri, J. N. Ruskin, and R. J. Cohen, Circulation 77, 110 (1988); B. Nearing, A. H. Huang, and R. L. Verrier, Science 252, 437 (1991); D. S. Rosenbaum, L. E. Jakson, J. M. Smith, H. Garam, J. N. Ruskin, and R. J. Cohen, N. Engl. J. Med. 330, 235 (1994).

[2] J. M. Pastore, S. D. Girouard, K. R. Laurita, F. G. Akar, and D. S. Rosenbaum, Circulation 99, 1385 (1999).

[3] Z. Qu, A. Garfinkel, P.-S. Chen, and J. N. Weiss, Circulation 102, 1664 (2000).

[4] M. A. Watanabe et al., J. Cardiovasc. Electrophysiol. 12, 196 (2001).

[5] M. Courtemanche, L. Glass, and J. P. Keener, Phys. Rev. Lett. 70, 2182 (1993).

[6] B. Echebarria and A. Karma, Phys. Rev. Lett. 88, 208101 (2002).

[7] B. Echebarria and A. Karma, Phys. Rev. E 76, 051911 (2007).

[8] J. B. Nolasco and R. W. Dahlen, J. Appl. Physiol 25, 191 (1968).

[9] M. R. Guevara, G. Ward, A. Shrier, and L. Glass, in Computers in Cardiology (IEEE Comp. Soc., New York, NY, USA, 1984), p. 167.
[10] J. J. Fox, E. Bodenschatz, and R. F. Gilmour, Phys. Rev. Lett. 89, 138101 (2002); E. G. Tolkacheva, D. G. Schaeffer, D. J. Gauthier, and W. Krassowska, Phys. Rev. E 67, 031904 (2003).

[11] Z. Qu, Y. Shiferaw, and J. N. Weiss, Phys. Rev. E 75, 011927 (2007).

[12] M. Watanabe, N. F. Otani, and R. F. Gilmour, Circ. Res. 76, 915 (1995).

[13] B. Echebarria, G. Röder, H. Engel, J. Davidsen, and M. Bär, Phys. Rev. E 83, 040902(R) (2011).

[14] A. V. Panfilov, R. H. Keldermann, and M. P. Nash, Proc. Natl. Acad. Sci. USA 104, 7922 (2007).

[15] H. Zhang, B.-W. Li, Z.-M. Sheng, Z. Cao, and G. Hu, Europhys. Lett. 76, 1109 (2006); H. Zhang, X. S. Ruan, B. Hu, and Q. Ouyang, Phys. Rev. E 70, 016212 (2004).

[16] See, e.g., the Special issue on Mechano-Electric Feedback and Cardiac Arrhythmias, P. Kohl and U. Ravens (eds.), Prog. Biophys. Mol. Biol. 82, 3 (2003).

[17] P. Kohl, P. Hunter, and D. Noble, Prog. Biophys. Mol. Biol. 71, 91 (1999). 
[18] P. J. Hunter, A. D. McCulloch, and H. E. D. J. ter Keurs, Prog. Biophys. Mol. Biol. 69, 289 (1998).

[19] M. P. Nash and A. V. Panfilov, Prog. Biophys. Mol. Biol. 85, 501 (2004).

[20] F. H. Fenton and A. Karma, Chaos 8, 20 (1998).

[21] E. Alvarez-Lacalle and B. Echebarria, Phys. Rev. E 79, 031921 (2009).

[22] Notice that the conductance of $J_{s a}$ in this formulation corresponds to $g_{s}=G_{s} k_{T} / E$ in Ref. [14], with $k_{T}$ being the normalization factor of the active tension $E=2\left(c_{1}+c_{2}\right)$ and $c_{1}$ and $c_{2}$ the coefficients of the strain energy function. Considering the parameters $k_{T}=5 \mathrm{MPa}, c_{1}=2 \mathrm{MPa}, c_{2}=6 \mathrm{MPa}$, then $G_{s}=3.2 g_{s}$.

[23] The parameters are $V_{0}=-85 \mathrm{mV}, V_{f i}=15 \mathrm{mV}, \tau_{d}=0.25 \mathrm{~ms}$, $\tau_{r}=33.3 \mathrm{~ms}, \quad \tau_{s i}=29.0 \mathrm{~ms}, \quad \tau_{0}=12.5 \mathrm{~ms}, \quad \tau_{v}^{+}=3.33 \mathrm{~ms}$, $\tau_{v 1}^{-}=1250 \mathrm{~ms}, \tau_{v 2}^{-}=19.6 \mathrm{~ms}, \tau_{w}^{+}=870 \mathrm{~ms}, \tau_{w}^{-}=41 \mathrm{~ms}, u_{c}=$ $0.13, \quad u_{v}=0.04, \quad u_{c}^{s i}=0.85, \quad E_{s}=1.0, \quad u_{T}=0.05, \quad \tau_{T 1}=$ $10.0 \mathrm{~ms}, \tau_{T 2}=1.0 \mathrm{~ms}, D=0.001 \mathrm{~cm}^{2} / \mathrm{ms}$.

[24] G. W. Beeler and H. Reuter, J. Physiol. (London) 268, 177 (1977).

[25] L. Weise and A. Panfilov, PLoS ONE 8, e59317 (2013).

[26] G. C. L. Bett and F. Sachs, J. Membr. Biol. 173, 237 (2000); W. Niu and F. Sachs, Prog. Biophys. Mol. Biol. 82, 121 (2003).
[27] S. C. Calaghan, A. Belus, and E. White, Prog. Biophys. Mol. Biol. 82, 81 (2003).

[28] T. G. McNarya, K. Sohna, B. Taccardia, and F. B. Sachse, Prog. Biophys. Mol. Biol. 97, 383 (2008).

[29] R. W. Mills, S. M. Narayan, and A. D. McCulloch, Am. J. Physiol. Heart Circ. Physiol. 295, H1270 (2008).

[30] N. H. L. Kuijpers, H. M. M. ten Eikelder, P. H. M. Bovendeerd, S. Verheule, T. Arts, and P. A. J. Hilbers, Am. J. Physiol. Heart Circ. Physiol. 292, H2832 (2007).

[31] Y. Hu, V. Gurev, J. Constantino, J. D. Bayer, and N. A. Trayanova, PLoS ONE 8, e60287 (2013).

[32] E. Alvarez-Lacalle, J. F. Rodríguez, and B. Echebarria, Comput. Cardiol. 35, 189 (2008).

[33] A. V. Panfilov, R. H. Keldermann, and M. P. Nash, Phys. Rev. Lett. 95, 258104 (2005).

[34] L. D. Weise, M. P. Nash, and A. V. Panfilov, PLoS ONE 6, e21934 (2011).

[35] J. G. Restrepo and A. Karma, Phys. Rev. E 79, 030906(R) (2009).

[36] T. Y. Kim, S.-J. Woo, S.-M. Hwang, J. H. Hong, and K. J. Lee, Proc. Natl. Acad. Sci. USA 104, 11639 (2007).

[37] T. Cameron and J. Davidsen, Phys. Rev. E 86, 061908 (2012). 